\title{
Systematic Review: The Comparative Content Review of the Persian Participation Assessment Measures: A Systematic Review
}

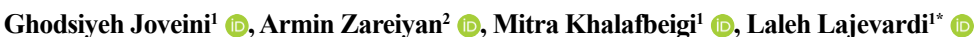

1. Department of Occupational Therapy, School of Rehabilitation Sciences, Iran University of Medical Sciences, Tehran, Iran

2. Department of Health in Disaster and Emergencies, Faculty of Nursing, AJA University of Medical Sciences, Tehran, Iran.

\begin{tabular}{|c|c|}
\hline $\begin{array}{l}\text { Use your device to scan } \\
\text { and read the article online }\end{array}$ & \\
\hline 口pra & $\begin{array}{l}\text { tion Assessment Measures: A Systematic Review Iranian Rehabilitation Journal. 2020; 18(1):1-16. http://dx.doi.org/10.32598/ } \\
\text { irj.18.1.928.1 }\end{array}$ \\
\hline 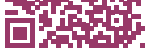 & doi http://dx.doi.org/10.32598/irj.18.1.928.1 \\
\hline
\end{tabular}

Article info:

Received: 15 Jul 2019

Accepted: 22 Oct 2019

Available Online: 01 Mar 2020

\section{Keywords:}

Participation, Assessment Measure, Persian, Systematic review

\section{ABSTRACT}

Objectives: Facilitating participation is regarded as the primary goal and outcome of rehabilitation. According to the importance of comprehensive assessment of participation in occupational areas (social participation, work, education, instrumental activities of daily living, activities of daily living, rest and sleep, leisure, and play), all available studies on the development or translation of measures, which assess participation in Persian, were systematically reviewed.

Methods: A search for Persian versions of measures, which assess participation in at least one occupational area, was carried out. Eight bibliographic databases, including 4 Iranian (IranDoc, Medlib, Magiran, and SID) and 4 international (Cochran, PubMed, Scopus, and ScienceDirect) databases from 1990-2018 were searched by 2 independent researchers based on the predetermined criteria. Any disagreements during the selection processes were resolved in consultation with the third researcher.

Results: Through 3 refining steps, by 2 independent researchers, 68 articles exactly related to this study were selected and studied. A total of 50 measures were extracted. The content of the measures and their psychometric properties were reported; 44 were translated into Persian and 6 developed based on the Iranian population.

Discussion: This critical review will help Persian-speaking therapists to select an appropriate measure for assessing participation in different occupational areas. Considering the importance of participation in the outcomes of rehabilitation and since it is mostly cultural and familialbased, developing new scales based on Persian-speaking people culture seems necessary.

* Corresponding Author:

Laleh Lajevardi, PhD.

Address: Department of Occupational Therapy, School of Rehabilitation Sciences, Iran University of Medical Sciences, Tehran, Iran Tel: +98 (21) 22228051

E-mail:laleh23275@yahoo.com 


\section{Highlights}

- Considering the lack of comprehensive measures for assessing participation in different ages, developing measures based on cultural properties seems necessary.

\section{Plain Language Summary}

Occupational therapy focuses on enabling people to participate in everyday occupations that are meaningful to them, giving them a sense of satisfaction, and leading them to their involvement in everyday life. Therapists should consider individual, cultural, and familial nature of the concept of participation and use appropriate assessments and interventions.

\section{Introduction}

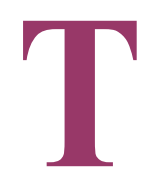

hrough participation in everyday occupations, people experience and master various skills, communicate with others, and find purpose and meaning of life [1] Engagement in meaningful occupations, which result in the well-being of people and their communities, is of importance. It is one of the human rights and it should be assured by equitable access to participation, regardless of differences [2]. The central focus of occupational therapy is promoting the health and wellbeing of people through occupation. Occupational therapy focuses on enabling people to participate in everyday occupations that are meaningful to them, giving them a sense of satisfaction, and leading them to their involvement in everyday life [3]. Enhancing participation in occupations is the key point.

The "Occupational Therapy Practice Framework: Domain and Process", an authorized essay of the American Occupational Therapy Association, provides a comprehensive view of different aspects of participation. According to the "Framework", participation in occupations consists of the following 8 life domains: social participation, work, education, Instrumental Activities Of Daily Living (IADLs), Activities Of Daily Living (ADLs), rest and sleep, leisure, and play [1].

Studies have shown that participation in various occupational areas has a significant role in the development of skills and health promotion [4-8]. On the other hand, the lack of participation or deprivation of meaningful and purposeful activities leads to a reduction in health and wellness [9].

Maintaining a satisfactory balance between these occupational areas, despite having a disability, is a determinant of health and well-being. One of the main goals of occupational therapy is achieving and sustaining occupational balance [10]. Accordingly, in occupational therapy, the accurate evaluation of daily occupations, in which people participate, is essential.

Concepts such as occupation are understood differently in various cultures. Besides, cultural expectations in disparate social contexts affect the type of activities that people do and the way they learn or perform them. For instance, research has shown that culture can have a significant influence on the level of participation in occupational areas. Thus, there are great differences among countries on this issue [11]

In recent years, many measures have developed to evaluate participation. The majority of participation measures have developed in foreign countries and often in the English language [12-14]. As a result, researchers interested in exploring this construct in a different language have had 2 options either to develop a new measure or to translate and use an existing one.

The aim of this study was to review all accessible resources thoroughly to collect and criticize the available assessment tools in Persian, which assess participation in at least one occupational area. This would be helpful for Persianspeaking occupational therapists in selecting an appropriate measure for assessing different aspects of the occupation.

\section{Methods}

Eight bibliographic databases, including 4 Iranian (IranDoc, Medlib, Magiran, and SID) and 4 international (Cochran, PubMed, Scopus, and ScienceDirect) databases were searched. The time of publication was limited from January 1, 1990, until the end of October 2018. Multiple combinations of keywords (with appropriate truncation) related to the construct (e,g., participation) and measure (e,g., Persian, questionnaire, and reliability) were used. 
Table 1. Search strategies used for searching PubMed databases

\begin{tabular}{cc}
\hline Database & Search strategies \\
& Search ("participation" [Title/ Abstract] OR “ADL" [Title/ Abstract] OR daily living activity [Title/ Abstract] OR play \\
[Title/ Abstract] OR leisure [Title/ Abstract] OR recreation *[Title/ Abstract] OR sleep [Title/ Abstract] OR rest [Title/ \\
Abstract] OR work [Title/ Abstract] OR productive activities [Title/ Abstract] OR education [Title/ Abstract] OR social \\
participation [Title/ Abstract]) AND (Farsi [Title/ Abstract] OR Persian [Title/ Abstract] OR Iran*[Title/ Abstract]) AND \\
(develop [Title/ Abstract] OR translation [Title/ Abstract] OR validity [Title/ Abstract] OR reliability [Title/ Abstract] OR \\
"psychometric properties" [Title/ Abstract] OR scale [Title/ Abstract] OR instrument [Title/ Abstract] OR tool [Title/ \\
Abstract] OR measure [Title/ Abstract] OR assessment [Title/ Abstract] OR questionnaire [Title/ Abstract] OR inven- \\
tory [Title/ Abstract]) Filters: Publication date from 1990/01/01 to 2018/12/31; Humans
\end{tabular}

Пranian Rehabilitation Journa

Table 1 presents two examples of how the articles were searched in Iranian and international databases. Search strategies in other databases are available from the authors on request.

Two researchers reviewed the title and abstracts independently. They assessed both generic and condition-specific measures that met the subsequent criteria: 1 . they had been developed or validated for use with Persian-speaking people; 2 . they seemed to cover items that assessed at least one domain of the "Framework"; 3. they existed in Persian; 4. there was information available on their psychometric properties; 5. their full text was accessible. At the next phase, full texts were looked over. References cited in retrieved articles were also searched and screened. Any disagreements during the selection processes were resolved in consultation with the third researcher.

\section{Results}

Figure 1 shows the procedure of searching databases, assortment, and the number of papers saved in each phase. Through 3 refining steps, 68 articles out of 3284 records were related to the present study.

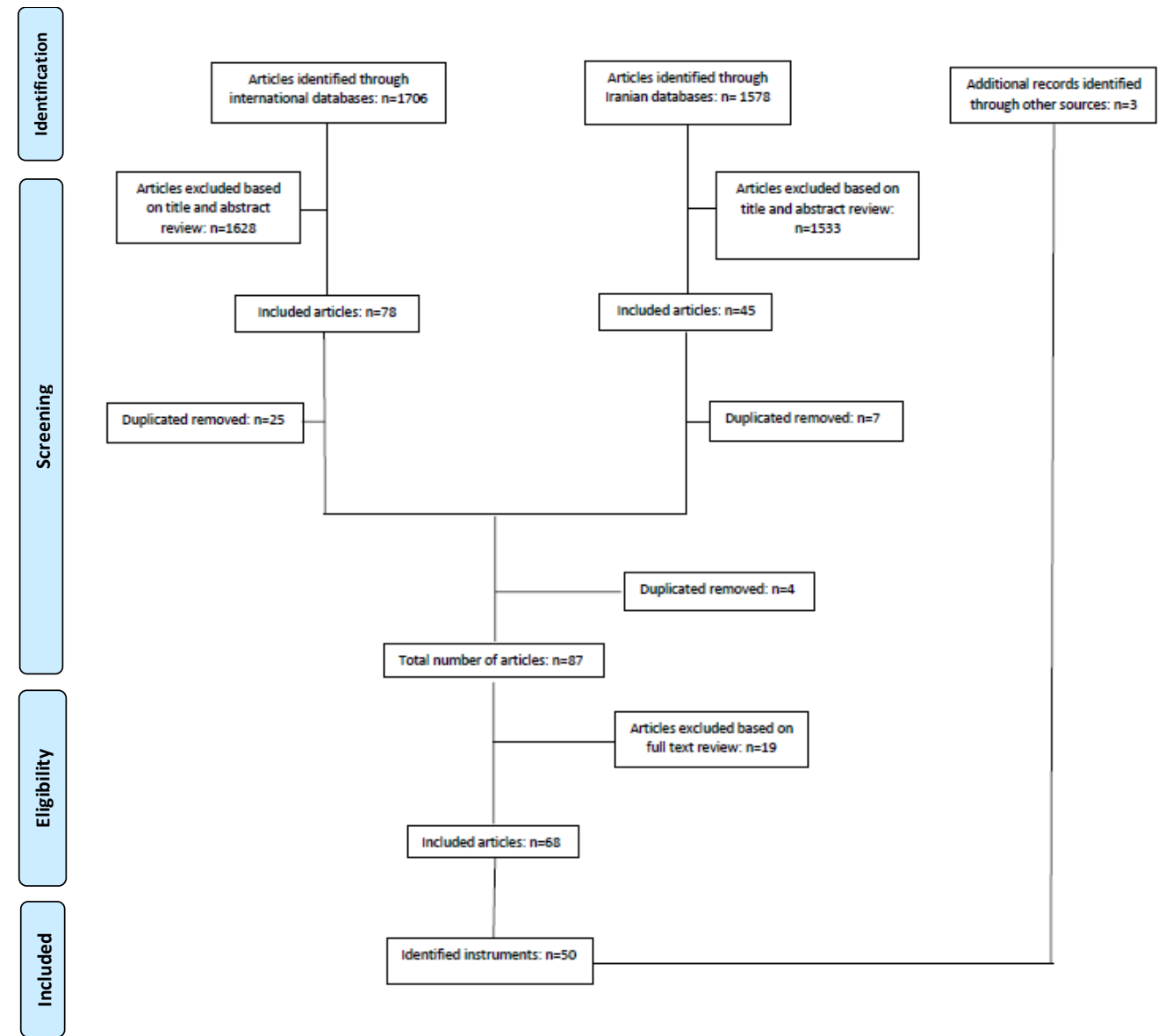

Figure 1. The process of searching 
Table 2. Participation measurements available in Persian

\begin{tabular}{|c|c|c|c|c|c|c|c|}
\hline No & Measure Name & 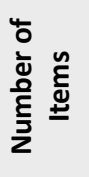 & Contents & 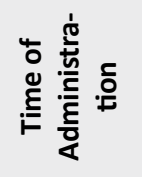 & 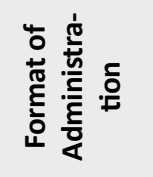 & 㟥 & 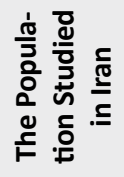 \\
\hline 1 & ADL and IADL scale [15] & 15 & $\begin{array}{l}\text { ADL (self-care, feeding, dressing, toilet } \\
\text { use, bowels, bladder control, transfer, mo- } \\
\text { bility, and bathing) and IADL (phone use, } \\
\text { drug use, meal preparation, household- } \\
\text { ing, shopping, money management, and } \\
\text { community mobility) }\end{array}$ & $10-15 \min$ & Interview & Anyone & $\begin{array}{l}\text { Geriat- } \\
\text { rics }>60 y\end{array}$ \\
\hline 2 & $\begin{array}{l}\text { Activities Scales for Kids } \\
\qquad[16]\end{array}$ & 30 & $\begin{array}{l}\text { Self-care, dressing, other skills, locomo- } \\
\text { tion, transfer, standing skills, and play }\end{array}$ & $15 \mathrm{~min}$ & $\begin{array}{l}\text { Child/par- } \\
\text { ent report }\end{array}$ & Anyone & $\begin{array}{l}\text { Cerebral } \\
\text { Palsy } \\
5-15 y\end{array}$ \\
\hline 3 & Barthel Index [17] & 10 & $\begin{array}{l}\text { Feeding, grooming, mobility, transfer, } \\
\text { stairs, dressing, bathing, toilet use, bow- } \\
\text { els, and bladder }\end{array}$ & $2-5 \min$ & Self-report & Anyone & Stroke \\
\hline 4 & $\begin{array}{l}\text { BEARS sleep screening tool } \\
{[18]}\end{array}$ & 5 & $\begin{array}{c}\text { Excessive daytime sleepiness, regularity, } \\
\text { duration of sleep and snoring, bedtime } \\
\text { problems, and awakenings during the } \\
\text { night }\end{array}$ & $5 \min$ & $\begin{array}{l}\text { Self-report } \\
\text { and parent } \\
\text { report }\end{array}$ & Anyone & $\begin{array}{l}\text { Children } \\
2-18 y\end{array}$ \\
\hline 5 & $\begin{array}{l}\text { Canadian Occupational } \\
\text { Performance Measure [19] }\end{array}$ & 9 & Self-care, productivity, and leisure & $20-30 \mathrm{~min}$ & Interview & $\begin{array}{l}\text { Trained } \\
\text { asses- } \\
\text { sor }\end{array}$ & $\begin{array}{l}\text { Mothers } \\
\text { of CP } \\
\text { children }\end{array}$ \\
\hline 6 & $\begin{array}{l}\text { Children's Assessment of } \\
\text { Participation and Enjoy- } \\
\text { ment [20] }\end{array}$ & 55 & Formal and informal leisure activities & $45-60 \mathrm{~min}$ & $\begin{array}{l}\text { Interview } \\
\text { or child } \\
\text { report }\end{array}$ & $\begin{array}{l}\text { Health } \\
\text { care } \\
\text { profes- } \\
\text { sional }\end{array}$ & $\begin{array}{l}\text { Disabled } \\
\text { children } \\
7-17 y\end{array}$ \\
\hline 7 & $\begin{array}{l}\text { Children's Sleep Habit } \\
\text { Questionnaire [21] }\end{array}$ & 45 & $\begin{array}{l}\text { Sleep onset delay, sleep anxiety, } \\
\text { parasomnia, bedtime resistance, sleep- } \\
\text { related breathing disorders and daytime } \\
\text { sleepiness, sleep duration, and awakening } \\
\text { during the night }\end{array}$ & $20-30 \mathrm{~min}$ & $\begin{array}{l}\text { Parent } \\
\text { report }\end{array}$ & Anyone & $\begin{array}{l}\text { Children } \\
7-12 y\end{array}$ \\
\hline 8 & $\begin{array}{l}\text { Child Initiated Pretend Play } \\
\text { Assessment }[22,23]\end{array}$ & 3 & $\begin{array}{l}\text { Percentage of elaborated pretend actions, } \\
\text { number of imitated action, number of } \\
\text { object substitution }\end{array}$ & $30 \mathrm{~min}$ & Interview & $\begin{array}{l}\text { Trained } \\
\text { asses- } \\
\text { sor }\end{array}$ & $\begin{array}{l}\text { Children } \\
4-7 y\end{array}$ \\
\hline 9 & $\begin{array}{l}\text { Children Participation } \\
\text { Questionnaire [24, 25] }\end{array}$ & 44 & $\begin{array}{l}\text { Play ،IADL, basic activities of daily living } \\
\text { ،education ،leisure, social participation }\end{array}$ & $15-20 \mathrm{~min}$ & $\begin{array}{l}\text { Parent } \\
\text { report }\end{array}$ & Anyone & $\begin{array}{l}\text { Children } \\
4-6 y\end{array}$ \\
\hline 10 & $\begin{array}{l}\text { Community Integration } \\
\text { Questionnaire [26] }\end{array}$ & 15 & $\begin{array}{l}\text { Home integration, social integration, and } \\
\text { integration into productive activities }\end{array}$ & $20-30 \mathrm{~min}$ & Self-report & Anyone & $\begin{array}{l}\text { Multiple } \\
\text { Sclerosis }\end{array}$ \\
\hline 11 & $\begin{array}{l}\text { Craig Hospital Inventory of } \\
\text { Environmental Factors [27] }\end{array}$ & 25 & $\begin{array}{c}\text { Services and assistance, structural, physi- } \\
\text { cal, school, policies, work, attitudes, and } \\
\text { support }\end{array}$ & $15 \mathrm{~min}$ & $\begin{array}{l}\text { Parent } \\
\text { report }\end{array}$ & Anyone & $\begin{array}{l}\text { Caregiv- } \\
\text { ers of CP } \\
\text { children }\end{array}$ \\
\hline 12 & $\begin{array}{l}\text { Epworth Sleepiness Scale } \\
\qquad[28]\end{array}$ & 8 & Sleepiness in 8 daily situations & 3-5 min & Self-report & Anyone & $\begin{array}{l}\text { Sleep dis- } \\
\text { orders }\end{array}$ \\
\hline 13 & $\begin{array}{l}\text { Functional Independence } \\
\text { Measure [29-31] }\end{array}$ & 18 & $\begin{array}{c}\text { Sphincter control, self-care, social cogni- } \\
\text { tion and communication, locomotion, and } \\
\text { mobility }\end{array}$ & $30-45 \min$ & Interview & $\begin{array}{l}\text { Trained } \\
\text { asses- } \\
\text { sor }\end{array}$ & Stroke \\
\hline 14 & $\begin{array}{l}\text { Global Sleep Assessment } \\
\text { [32] }\end{array}$ & 11 & Sleep behaviors & $5 \min$ & Self-report & Anyone & Nurses \\
\hline 15 & $\begin{array}{c}\text { Impact on Participation } \\
\text { and Autonomy Question- } \\
\text { naire }[33,34]\end{array}$ & 32 & $\begin{array}{c}\text { Self-care, money management, leisure, } \\
\text { mobility social relationships, paid work, } \\
\text { education, learning, living as a life hole, } \\
\text { and home management }\end{array}$ & $20-30 \mathrm{~min}$ & Interview & $\begin{array}{l}\text { Health } \\
\text { care } \\
\text { profes- } \\
\text { sional }\end{array}$ & $\begin{array}{l}\text { Stroke } \\
\text { and } \\
\text { multiple } \\
\text { sclerosis }\end{array}$ \\
\hline 16 & $\begin{array}{l}\text { Independence Scale of Ac- } \\
\text { tivities of Daily Living [35] }\end{array}$ & 20 & ADL & $10-15 \min$ & Interview & $\begin{array}{l}\text { Health } \\
\text { care } \\
\text { profes- } \\
\text { sional }\end{array}$ & Stroke \\
\hline
\end{tabular}




\begin{tabular}{|c|c|c|c|c|c|c|c|}
\hline No & Measure Name & 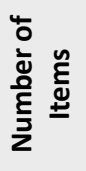 & Contents & 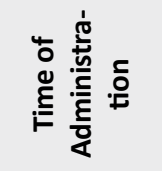 & 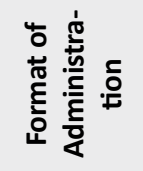 & 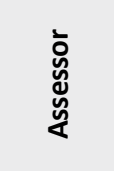 & 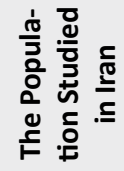 \\
\hline 17 & $\begin{array}{l}\text { Insomnia Severity Index } \\
\text { [36] }\end{array}$ & 19 & $\begin{array}{l}\text { Difficulty at the beginning of sleep, staying } \\
\text { asleep, waking up too early, satisfaction } \\
\text { with sleep, concern caused by sleep prob- } \\
\text { lems, interference with daytime function- } \\
\text { ing, and noticeability of impairment }\end{array}$ & $5 \mathrm{~min}$ & Self-report & Anyone & $\begin{array}{l}\text { Sleep } \\
\text { clinic } \\
\text { patients }\end{array}$ \\
\hline 18 & $\begin{array}{c}\text { Iranian Children's Participa- } \\
\text { tion Assessment Scale } \\
{[37-39]}\end{array}$ & 71 & $\begin{array}{l}\text { Work, play, leisure, social participation, } \\
\text { education, ADL, IADL, and sleep/rest }\end{array}$ & $\begin{array}{l}45-60 \text { min } \\
\text { for chil- } \\
\text { dren } / 30-45 \\
\text { min for } \\
\text { parents }\end{array}$ & $\begin{array}{l}\text { Child } \\
\text { report and } \\
\text { parent } \\
\text { report }\end{array}$ & Anyone & $\begin{array}{l}\text { Children } \\
6-12 y\end{array}$ \\
\hline 19 & $\begin{array}{l}\text { Iranian male adolescents } \\
\text { outcome expectation } \\
\text { about leisure time physical } \\
\text { activity [40] }\end{array}$ & 26 & $\begin{array}{l}\text { Self-evaluation, social expectancy, and } \\
\text { physical expectancy }\end{array}$ & $10-15 \min$ & Self-report & Anyone & $15-19 y$ \\
\hline 20 & $\begin{array}{l}\text { International Physical Activ- } \\
\text { ity Questionnaire }[41,42]\end{array}$ & 25 & $\begin{array}{l}\text { Occupational, transportation, household/ } \\
\text { gardening, and leisure time activities }\end{array}$ & $10-15 \min$ & Self-report & Anyone & Adults \\
\hline 21 & $\begin{array}{l}\text { Job Content Questionnaire } \\
\text { [43] }\end{array}$ & 39 & $\begin{array}{l}\text { Decision authority, skill dissertation, } \\
\text { psychological demand, physical excretion, } \\
\text { job insecurity, physical trauma, supervisor } \\
\text { support, coworkers support, job satisfac- } \\
\text { tion, anxiety, and depression scales }\end{array}$ & $15 \mathrm{~min}$ & Self-report & Anyone & $\begin{array}{l}\text { Iranian } \\
\text { health } \\
\text { care } \\
\text { workers }\end{array}$ \\
\hline 22 & KATZ [44] & 6 & $\begin{array}{l}\text { Continence, dressing, using the bathroom, } \\
\text { getting up and being able to move around } \\
\text { the house, feeding, and bathing }\end{array}$ & $5-10 \mathrm{~min}$ & Self-report & Anyone & $\begin{array}{c}\text { Stroke } \\
18-81 y \\
\text { elderly } \\
\text { patients } \\
\text { with } \\
\text { cancer } \\
>60 y\end{array}$ \\
\hline 23 & Lawton IADL scale $[45,46]$ & 8 & $\begin{array}{c}\text { Using the telephone, shopping, preparing } \\
\text { food, housekeeping, doing laundry, using } \\
\text { transportation, handling medications, and } \\
\text { handling finances }\end{array}$ & $5-10 \mathrm{~min}$ & Self-report & Anyone & $\begin{array}{l}\text { Geriat- } \\
\text { rics with } \\
\text { demen- } \\
\text { tia } 60 y>\end{array}$ \\
\hline 24 & $\begin{array}{l}\text { Assessment of Life Habits } \\
\text { [47] }\end{array}$ & 77 & Daily activities and social roles & 20-30 min & Self-report & Anyone & $\begin{array}{c}\text { Geriatrics } \\
60 y>C P \\
3-15 y\end{array}$ \\
\hline 25 & $\begin{array}{l}\text { Modifiable Activity Ques- } \\
\text { tionnaire }[48,49]\end{array}$ & 40 & $\begin{array}{l}\text { Leisure time and occupational physical } \\
\text { activity }\end{array}$ & $5-10 \mathrm{~min}$ & Self-report & Anyone & $\begin{array}{l}\text { Adults } \\
>19 y \\
\text { Adoles- } \\
\text { cents } \\
12-18 y\end{array}$ \\
\hline 26 & $\begin{array}{l}\text { Modified Barthel Index } \\
\qquad[50]\end{array}$ & 10 & $\begin{array}{l}\text { Toilet use, feeding, grooming, mobility, } \\
\text { dressing transfer, stairs, and bowels bath- } \\
\text { ing bladder }\end{array}$ & $2-5 \min$ & Interview & Anyone & Elderly \\
\hline 27 & $\begin{array}{l}\text { Occupational Gaps Ques- } \\
\text { tionnaire }[51]\end{array}$ & 31 & IADL, leisure, social activities, and work & $10-15 \min$ & Self-report & Anyone & $\begin{array}{l}\text { Patients } \\
\text { with } \\
\text { stroke }\end{array}$ \\
\hline 28 & $\begin{array}{l}\text { Onyx Social Capital Scale } \\
\qquad[52]\end{array}$ & 7 & $\begin{array}{c}\text { Social cooperation, trust feeling, life } \\
\text { worthwhile, work relations, accepting } \\
\text { differences, family relationships, and local } \\
\text { solidarity }\end{array}$ & $5-10 \min$ & Self-report & Anyone & $\begin{array}{c}\text { Geriatrics } \\
60 y>\end{array}$ \\
\hline 29 & $\begin{array}{l}\text { Performance Assessment } \\
\text { of Self-care Skills [53] }\end{array}$ & 24 & ADL, IADL, and mobility & $10-15 \min$ & Self-report & Anyone & $\begin{array}{l}\text { Multiple } \\
\text { Sclerosis }\end{array}$ \\
\hline 30 & $\begin{array}{l}\text { Pediatric Evaluation of Dis- } \\
\text { ability Inventory [54] }\end{array}$ & 197 & Self-care, mobility, and social performance & $30-45 \min$ & $\begin{array}{l}\text { Interview, } \\
\text { parent } \\
\text { report, or } \\
\text { both }\end{array}$ & $\begin{array}{l}\text { Health } \\
\text { care } \\
\text { profes- } \\
\text { sional }\end{array}$ & $\begin{array}{l}\text { Child 0.5- } \\
7.5 y\end{array}$ \\
\hline
\end{tabular}




\begin{tabular}{|c|c|c|c|c|c|c|c|}
\hline No & Measure Name & 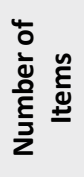 & Contents & 늘 & 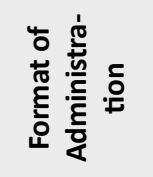 & 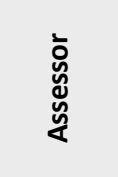 & 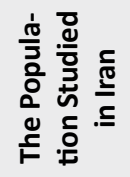 \\
\hline 31 & $\begin{array}{l}\text { Pittsburg Sleep Quality } \\
\text { Index [55] }\end{array}$ & 19 & $\begin{array}{l}\text { Subjective sleep quality, sleep latency, } \\
\text { sleep duration, habitual sleep efficiency, } \\
\text { sleep disturbances, use of sleeping medi- } \\
\text { cation, and daytime medication in the } \\
\text { previous month }\end{array}$ & $10 \mathrm{~min}$ & Self-report & Anyone & $\begin{array}{l}\text { Patients } \\
\text { with } \\
\text { sleep } \\
\text { problems }\end{array}$ \\
\hline 32 & $\begin{array}{l}\text { Pittsburgh Sleep Quality } \\
\text { Index Addendum [56] }\end{array}$ & 7 & Disruptive nocturnal behaviors & $5 \mathrm{~min}$ & Self-report & Anyone & $\begin{array}{l}\text { Patients } \\
\text { with } \\
\text { post- } \\
\text { traumat- } \\
\text { ic stress } \\
\text { disorder }\end{array}$ \\
\hline 33 & $\begin{array}{l}\text { Quantification de l'Activite } \\
\text { Physique en Altitude Chez } \\
\text { les Enfants [57] }\end{array}$ & 12 & $\begin{array}{l}\text { Home activities, daily activities (meals, } \\
\text { toilet, transportation, and sleeping), } \\
\text { activities during school period (sport com- } \\
\text { petition, training out of school activities, } \\
\text { vacation activities, and personal artistic } \\
\text { activities not associated with school), and } \\
\text { physical activity at school (mandatory } \\
\text { physical education, activities in classroom, } \\
\text { and other activities) }\end{array}$ & $5-10 \min$ & Self-report & Anyone & $\begin{array}{l}\text { Adoles- } \\
\text { cents } \\
15-18\end{array}$ \\
\hline 34 & $\begin{array}{l}\text { Rheumatoid and Arthritis } \\
\text { Outcome Score [58] }\end{array}$ & 42 & $\begin{array}{l}\text { Pain, symptoms, ADL, sport and recre- } \\
\text { ation, and quality of life }\end{array}$ & $20-30 \mathrm{~min}$ & Self-report & Anyone & $\begin{array}{l}\text { Rheu- } \\
\text { matoid } \\
\text { arthritis }\end{array}$ \\
\hline 35 & $\begin{array}{l}\text { Recreation Experience } \\
\text { Preference Scale [59] }\end{array}$ & 17 & $\begin{array}{l}\text { Success, nature enjoyment, loneliness, } \\
\text { socialization, and health }\end{array}$ & $5-10 \min$ & Self-report & Anyone & $\begin{array}{l}\text { Geriatrics } \\
60 y>\end{array}$ \\
\hline 36 & Role Strain Index [60] & 44 & $\begin{array}{l}\text { Inter-role conflict, inter- and intra-sender } \\
\text { conflict role overload, incongruity, incom- } \\
\text { petence, and ambiguity }\end{array}$ & $10-15 \min$ & Self-report & Anyone & $\begin{array}{l}\text { Nursing } \\
\text { teacher }\end{array}$ \\
\hline 37 & $\begin{array}{l}\text { Self-efficacy about leisure } \\
\text { time physical activity [61] }\end{array}$ & 13 & $\begin{array}{l}\text { Overcoming barriers, program adjust- } \\
\text { ment, and implementation of programs }\end{array}$ & $5 \mathrm{~min}$ & Self-report & Anyone & $\begin{array}{l}\text { Male } \\
\text { adoles- } \\
\text { cents } \\
15-19 y\end{array}$ \\
\hline 38 & $\begin{array}{l}\text { Self-regulation about } \\
\text { leisure time physical activ- } \\
\text { ity [62] }\end{array}$ & 16 & $\begin{array}{l}\text { Enlistment of social support, goal setting, } \\
\text { self-construction, and self-monitoring }\end{array}$ & $5-10 \min$ & Self-report & Anyone & $\begin{array}{l}\text { Male } \\
\text { adoles- } \\
\text { cents } \\
15-19 y\end{array}$ \\
\hline 39 & $\begin{array}{l}\text { Sleep Disturbance Scale for } \\
\text { Children [63] }\end{array}$ & 26 & $\begin{array}{l}\text { Disorder of initiating and maintaining } \\
\text { sleep, sleep-disordered breathing, the } \\
\text { disorder of arousal, sleep-wake transition } \\
\text { disorder, disorders of excessive somno- } \\
\text { lence, and sleep hyperhidrosis }\end{array}$ & $10-15 \mathrm{~min}$ & $\begin{array}{l}\text { Caregiver } \\
\text { report }\end{array}$ & Anyone & $\begin{array}{l}\text { Children } \\
6-15 y\end{array}$ \\
\hline 40 & Sleep Hygiene Index [64] & 13 & $\begin{array}{l}\text { Sleep-wake cycle, bedroom, and behav- } \\
\text { iors affecting sleep }\end{array}$ & $10-15 \mathrm{~min}$ & $\begin{array}{l}\text { Caregiver } \\
\text { report }\end{array}$ & Anyone & $\begin{array}{l}\text { Women } \\
20-60 y / \\
\text { general } \\
\text { popula- } \\
\text { tion }\end{array}$ \\
\hline 41 & $\begin{array}{c}\text { Adolescent Sleep Hygiene } \\
\text { Scale [65] }\end{array}$ & 28 & $\begin{array}{l}\text { Nine domains of sleep hygiene prac- } \\
\text { tices include physiological, cognitive, } \\
\text { emotional, sleep environment, daytime } \\
\text { sleep, substances, sleep stability, bedtime } \\
\text { routine, and bed-sharing factor }\end{array}$ & $10-15 \min$ & Self-report & Anyone & $\begin{array}{l}\text { Adoles- } \\
\text { cents } \\
12-19 y\end{array}$ \\
\hline 42 & $\begin{array}{l}\text { Specific Nordic Question- } \\
\text { naire [66] }\end{array}$ & 40 & Work-related musculoskeletal disorders & $10-15$ & Self-report & Anyone & $\begin{array}{l}\text { Industrial } \\
\text { workers }\end{array}$ \\
\hline 43 & $\begin{array}{l}\text { Task Self-Efficacy Scale for } \\
\text { Everyday Activities [67] }\end{array}$ & 18 & Self-care & $10 \mathrm{~min}$ & Self-report & Anyone & $\begin{array}{l}\text { Geriatrics } \\
60 y>\end{array}$ \\
\hline 44 & $\begin{array}{l}\text { Social Participation Ques- } \\
\text { tionnaire [68] }\end{array}$ & 21 & Social participation & $10 \mathrm{~min}$ & Self-report & Anyone & $\begin{array}{l}\text { Adoles- } \\
\text { cents } \\
15-18 y\end{array}$ \\
\hline
\end{tabular}




\begin{tabular}{|c|c|c|c|c|c|c|c|}
\hline No & Measure Name & 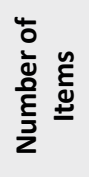 & Contents & 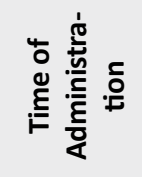 & 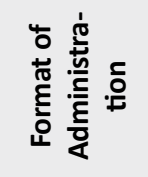 & 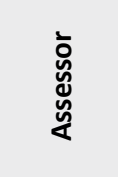 & 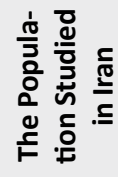 \\
\hline 45 & $\begin{array}{l}\text { Verran and Snyder-Halpern } \\
\text { Sleep Scale [69] }\end{array}$ & 11 & $\begin{array}{l}\text { Disturbance, effectiveness, and supple- } \\
\text { mentation }\end{array}$ & $5-10 \mathrm{~min}$ & Self-report & Anyone & $\begin{array}{l}\text { Hospi- } \\
\text { talized } \\
\text { patients }\end{array}$ \\
\hline 46 & $\begin{array}{l}\text { Walton's quality of work } \\
\text { life [70] }\end{array}$ & 24 & $\begin{array}{l}\text { Opportunity to use and develop human } \\
\text { capacities and continued growth and } \\
\text { security, adequate and fair compensa- } \\
\text { tion, safe and healthy working conditions, } \\
\text { constitutionalization in the work organiza- } \\
\text { tion, work and total life span, and social } \\
\text { relevance of work life social integration in } \\
\text { the work organization }\end{array}$ & $10-15 \min$ & Self-report & Anyone & Nurses \\
\hline 47 & $\begin{array}{c}\text { Wheelchair Outcome } \\
\text { Measure [71] }\end{array}$ & 6 & $\begin{array}{l}\text { Part one: importance and satisfaction } \\
\text { of participation goals in home and com- } \\
\text { munity, } \\
\text { Part two: } 3 \text { structured questions; the } \\
\text { client's comfort while sitting in the wheel- } \\
\text { chair, satisfaction with body positioning in } \\
\text { the wheelchair, and any experience of skin } \\
\text { breakdown over the past month }\end{array}$ & $20-30 \mathrm{~min}$ & Interview & Anyone & $\begin{array}{l}\text { Patients } \\
\text { with spi- } \\
\text { nal cord } \\
\text { injury }\end{array}$ \\
\hline 48 & $\begin{array}{l}\text { Work Ability Index Ques- } \\
\text { tionnaire [72] }\end{array}$ & 10 & $\begin{array}{c}\text { Current work ability regarding the de- } \\
\text { mands of the job compared with lifetime } \\
\text { best, current disease and estimated work } \\
\text { impairment, personal prediction of work } \\
\text { ability } 2 \text { years from now, and mental } \\
\text { properties sick leave during the past } 12 \\
\text { months }\end{array}$ & $5-10 \mathrm{~min}$ & Self-report & Anyone & $\begin{array}{l}\text { Iranian } \\
\text { workers }\end{array}$ \\
\hline 49 & $\begin{array}{l}\text { World Health Organization } \\
\text { Health and Work Perfor- } \\
\text { mance Questionnaire [73] }\end{array}$ & 91 & $\begin{array}{l}\text { Work (hours, sick leaves, occupational } \\
\text { accidents and self-rated output in past } 7 \\
\text { days and past } 4 \text { weeks) and health (physi- } \\
\text { cal health status, mental health, recent } \\
\text { physical health status, recent mental } \\
\text { health status, and history of medical visits } \\
\text { in the past year) }\end{array}$ & $20-30 \mathrm{~min}$ & Self-report & Anyone & $\begin{array}{l}\text { Iranian } \\
\text { health } \\
\text { care } \\
\text { workers }\end{array}$ \\
\hline 50 & $\begin{array}{l}\text { Work-related Low Back } \\
\text { Pain Predictor Question- } \\
\text { naire [74] }\end{array}$ & 40 & $\begin{array}{l}\text { Self-control, emotional coping, knowl- } \\
\text { edge, outcome perception, and self-effica- } \\
\text { cy in overcoming impediments and chal- } \\
\text { lenges in the environment self-efficacy }\end{array}$ & $15-20 \mathrm{~min}$ & Self-report & Anyone & $\begin{array}{l}\text { Patients } \\
\text { care } \\
\text { workers } \\
>18 y\end{array}$ \\
\hline
\end{tabular}

Table 3. Psychometric properties of Persian participation measures

\begin{tabular}{|c|c|c|c|c|}
\hline No & Measure & $\begin{array}{l}\text { Occupational Therapy Practice } \\
\text { Framework Dimensions }\end{array}$ & Validity & Reliability \\
\hline 1 & $\begin{array}{l}\text { Activities of Daily Living } \\
\text { (ADL) and Instrumental } \\
\text { Activities of Daily } \\
\text { Living (IADL) scale }\end{array}$ & ADL1 and IADL2 & $\begin{array}{l}\text { Content Validity Index (CVI): } \\
\text { (ADL: } 0.82 \text {, IADL: } 0.85 \text { ) } \\
\text { Convergent validity: } P<0.001\end{array}$ & $\begin{array}{l}\text { ICC: (ADL: 0.76, IADL: 0.79) } \\
\text { Internal Consistency: (ADL: } \\
\quad 0.8, \text { IADL: } 0.75 \text { ) }\end{array}$ \\
\hline 2 & $\begin{array}{l}\text { Activities scales for } \\
\text { kids }\end{array}$ & $\mathrm{ADL}$ and leisure & $\begin{array}{c}\text { CVI: } 0.79-0.86 \\
\text { Convergent validity: } P<0.001\end{array}$ & $\begin{array}{c}\text { ICC }=0.99 \\
\text { Internal Consistency }=0.997\end{array}$ \\
\hline 3 & Barthel index & $A D L$ & $\begin{array}{l}\text { Concurrent validity in stroke: } \\
r=-0.912\end{array}$ & $\begin{array}{c}\text { ICC }=0.98 \\
\text { Internal consistency }>0.9\end{array}$ \\
\hline 4 & $\begin{array}{l}\text { BEARS sleep screen- } \\
\text { ing tool }\end{array}$ & Sleep/Rest & Criteria validity: good $\mathrm{P}<0.05$ & ICC: good to excellent $P<0.05$ \\
\hline 5 & $\begin{array}{l}\text { Canadian occupational } \\
\text { performance measure }\end{array}$ & $A D L, I A D L$, and leisure & $\begin{array}{c}\text { Content Validity Ratio (CVR): } \\
80.95-0.222\end{array}$ & $r: 0.84-0.87$ \\
\hline 6 & $\begin{array}{l}\text { Children's assessment } \\
\text { of participation and } \\
\text { enjoyment }\end{array}$ & $\begin{array}{c}\text { Play, leisure, and social participa- } \\
\text { tion }\end{array}$ & $\begin{array}{l}\text { CVI }=0.75 \\
\text { Discriminate validity: } 85 \%\end{array}$ & $\begin{array}{l}\text { ICC: } 0.44-0.89 \\
\text { Internal consistency }=0.86\end{array}$ \\
\hline
\end{tabular}




\begin{tabular}{|c|c|c|c|c|}
\hline No & Measure & $\begin{array}{l}\text { Occupational Therapy Practice } \\
\text { Framework Dimensions }\end{array}$ & Validity & Reliability \\
\hline 7 & $\begin{array}{l}\text { Children's sleep habit } \\
\text { questionnaire }\end{array}$ & Sleep/Rest & $\begin{array}{l}\text { Convergence validity: } 0.4 \text { to } \\
0.86 \\
\text { Divergence validity: } 0.006 \\
\text { to } 0.66\end{array}$ & Acceptable \\
\hline 8 & $\begin{array}{l}\text { Child initiated pretend } \\
\text { play assessment }\end{array}$ & Play & $C V I=1$ & - \\
\hline 9 & $\begin{array}{l}\text { Children participation } \\
\text { questionnaire }\end{array}$ & $\begin{array}{l}A D L, I A D L \text {, play, leisure, social } \\
\text { participation, and education }\end{array}$ & $\begin{array}{c}\mathrm{CV} />0.79 \\
\text { Convergent validity: }(r>0.66, \\
\mathrm{P}<(0.001)\end{array}$ & - \\
\hline 10 & $\begin{array}{l}\text { Community integration } \\
\text { questionnaire }\end{array}$ & $\begin{array}{c}\text { IADL, leisure, social participation, } \\
\text { and work }\end{array}$ & $\begin{array}{l}\text { Construct validity: }(r=0.40 \\
\qquad<<0.001)\end{array}$ & $\begin{array}{l}\text { ICC }>0.70 \\
\text { Internal consistency }>0.70 \\
\text { only for home integration } \\
\text { items }\end{array}$ \\
\hline 11 & $\begin{array}{l}\text { Craig hospital inven- } \\
\text { tory of environmental } \\
\text { factors }\end{array}$ & $\begin{array}{l}\text { ADL, leisure, social participation, } \\
\text { education, and work }\end{array}$ & $\begin{array}{c}\text { Construct validity: moderate } \\
r=0.21-0.47 \\
\text { Discriminate validity }>0.40\end{array}$ & $\begin{array}{c}I C C>0.70 \\
\text { Internal consistency }=0.86\end{array}$ \\
\hline 12 & $\begin{array}{l}\text { Epworth sleepiness } \\
\text { scale }\end{array}$ & Sleep/Rest & $\begin{array}{c}\text { All fit indices acceptable } 6 \\
\text { Criterion validity: } r=0.21-0.27 \\
P<0.05\end{array}$ & $\begin{array}{c}\text { ICC }=0.81 \\
\text { Internal consistency }=0.82\end{array}$ \\
\hline 13 & $\begin{array}{l}\text { Functional indepen- } \\
\text { dence measure }\end{array}$ & ADL and social participation & $\begin{array}{l}\text { Construct validity: }(r=0.95 \\
\qquad P<0.001)\end{array}$ & $\begin{array}{c}\mathrm{ICC}=0.88-0.98 \\
\text { Internal consisten- } \\
\mathrm{Cy}=0.70-0.96\end{array}$ \\
\hline 14 & $\begin{array}{l}\text { Global sleep assess- } \\
\text { ment }\end{array}$ & Sleep/Rest & $\begin{array}{c}\text { Content validity }>0.70 \\
\text { Concurrent validity: }(r>0.76, \\
P<(0.001)\end{array}$ & $\begin{array}{c}\mathrm{ICC}=0.86 \\
\text { Internal consistency }=0.87\end{array}$ \\
\hline 15 & $\begin{array}{l}\text { Impact on participa- } \\
\text { tion and autonomy } \\
\text { questionnaire }\end{array}$ & $\begin{array}{l}\text { ADL, IADL, leisure, social participa- } \\
\text { tion, education, and work }\end{array}$ & All fit indices acceptable & Person reliability $=0.92$ \\
\hline 16 & $\begin{array}{l}\text { Insomnia severity } \\
\text { index }\end{array}$ & Sleep/Rest & $\begin{array}{l}\text { Concurrent validity: }(r>0.74, \\
P<0.001)\end{array}$ & Internal consistency $=0.78$ \\
\hline 17 & $\begin{array}{l}\text { Iranian children's par- } \\
\text { ticipation assessment } \\
\text { scale }\end{array}$ & Sleep/Rest & $\begin{array}{l}\text { CVI>0.79 } \\
\text { All fit indices acceptable }\end{array}$ & $\begin{array}{l}\mathrm{ICC}>0.7 \text { in child report, }>0.8 \\
\text { in parent report }\end{array}$ \\
\hline 18 & $\begin{array}{l}\text { Iranian male adoles- } \\
\text { cents outcome expec- } \\
\text { tation about leisure } \\
\text { time physical activity }\end{array}$ & Leisure & All fit indices acceptable & Internal consistency $=0.85$ \\
\hline 19 & $\begin{array}{l}\text { Independence scale of } \\
\text { activities of daily living }\end{array}$ & ADL and IADL & Content validity $>0.70$ & Internal consistency $=0.98$ \\
\hline 20 & $\begin{array}{l}\text { International physical } \\
\text { activity questionnaire }\end{array}$ & ADL, IADL, leisure, and work & $\begin{array}{l}\text { Discriminate validity: }(r=0.26 \\
\qquad P<0.001)\end{array}$ & ICC $>0.70$ \\
\hline 21 & $\begin{array}{l}\text { Job content question- } \\
\text { naire }\end{array}$ & Work & All fit indices acceptable & $\begin{array}{c}\text { K statistics: } 0.60-0.80 \\
\text { Internal consistency }>0.75 \\
\text { except for psychological } \\
\text { demand }(\alpha=0.60) \text { and job } \\
\text { insecurity }(\alpha=0.27)\end{array}$ \\
\hline 22 & KATZ & ADL & $\begin{array}{l}\text { Criterion validity: ( } r=0.572, \\
P>001 \text { in cancerous geriatrics) }\end{array}$ & $\begin{array}{c}\text { ICC: } 0.78 \text { in stoke, }(r=0.83 \\
P>001 \text { in cancerous geriat- } \\
\text { rics) } \\
\text { Internal consistency=0.81 } \\
\text { in stroke, (0.92 in cancerous } \\
\text { geriatrics) }\end{array}$ \\
\hline
\end{tabular}




\begin{tabular}{|c|c|c|c|c|}
\hline No & Measure & $\begin{array}{l}\text { Occupational Therapy Practice } \\
\text { Framework Dimensions }\end{array}$ & Validity & Reliability \\
\hline 23 & Lawton IADL scale & IADL & $\begin{array}{l}\text { Construct validity: } x 2=19.02 \\
\qquad \mathrm{P}<0.05\end{array}$ & $\begin{array}{c}\text { Test-retest with relative } \\
\text { and absolute coefficients: } \\
(r=0.99 \text { and SEM=0.23) }(\mathrm{Cl} \text { : } \\
0.98-0.99) \text {. Correlation coef- } \\
\text { ficients between the raters } \\
(r=0.96)\end{array}$ \\
\hline 24 & $\begin{array}{l}\text { Assessment of life } \\
\text { habits }\end{array}$ & ADL and social participation & $\begin{array}{l}\text { CVI: } 0.88 \text { in geriatrics } \\
\text { Content validity: } r>0.50 \\
P>0.001 \text { in } C P \text { children }\end{array}$ & $\begin{array}{c}\text { ICC: (0.95 in geriatrics), }(0.78 \\
\text { in CP children) }\end{array}$ \\
\hline 25 & $\begin{array}{l}\text { Modifiable activity } \\
\text { questionnaire }\end{array}$ & Leisure and work & $\begin{array}{l}\text { Convergent validity: (In } \\
\text { adults: } r=0.47 \mathrm{P}<0.001 \text { ) } \\
\text { (In adolescents: } r=0.49 \text {, } \\
\quad P<0.001 \text { ) }\end{array}$ & $\begin{array}{l}\text { ICC: (In adults: 0.94), (In } \\
\text { adolescents: 0.97) }\end{array}$ \\
\hline 26 & Modified barthel index & $A D L$ & $\begin{array}{l}\text { Concurrent validity: }(r=0.993, \\
\qquad P<0.001)\end{array}$ & $\begin{array}{c}\mathrm{ICC}=0.99 \\
\text { Internal consistency: 0.96- } \\
0.99\end{array}$ \\
\hline 27 & $\begin{array}{l}\text { Occupational gaps } \\
\text { questionnaire }\end{array}$ & $\begin{array}{l}\text { IADL, leisure, social participation, } \\
\text { and work }\end{array}$ & All fit indices acceptable & - \\
\hline 28 & $\begin{array}{l}\text { Onyx social capital } \\
\text { scale }\end{array}$ & Social participation & All fit indices acceptable & $\begin{array}{c}\text { ICC }>0.7 \\
\text { Internal consistency: } 0.96\end{array}$ \\
\hline 29 & $\begin{array}{l}\text { Performance assess- } \\
\text { ment of self-care skills }\end{array}$ & ADL and IADL & Content validity: $91-94 \%$ & - \\
\hline 30 & $\begin{array}{l}\text { Pediatric evaluation of } \\
\text { disability inventory }\end{array}$ & ADL and social participation & All fit indices acceptable & - \\
\hline 31 & $\begin{array}{l}\text { Pittsburg sleep quality } \\
\text { index }\end{array}$ & Sleep/Rest & All fit indices acceptable & Internal consistency=0.55 \\
\hline 32 & $\begin{array}{l}\text { Pittsburgh sleep qual- } \\
\text { ity index addendum }\end{array}$ & Sleep/Rest & $\begin{array}{l}\text { Construct validity: } r=0.66 \\
\qquad(P<0.001)\end{array}$ & Internal consistency=0.88 \\
\hline 33 & $\begin{array}{c}\text { Quantification de } \\
\text { l'Activite Physique } \\
\text { en Altitude Chez les } \\
\text { Enfants }\end{array}$ & Sleep/Rest & All fit indices acceptable & ICC: $0.79-0.98$ \\
\hline 34 & $\begin{array}{l}\text { Rheumatoid and ar- } \\
\text { thritis outcome score }\end{array}$ & ADL and Leisure & All fit indices acceptable & $\begin{array}{c}\text { ICC }>0.70 \\
\text { Internal consistency }>0.70\end{array}$ \\
\hline 35 & $\begin{array}{l}\text { Recreation experience } \\
\text { preference scale }\end{array}$ & Leisure & All fit indices acceptable & $\begin{array}{l}\text { Internal consistency: } 0.7- \\
0.92\end{array}$ \\
\hline 36 & Role strain index & Work & CVI $>0.80$ & $\begin{array}{c}\mathrm{ICC}=0.91 \\
\text { Internal consistency }=0.92\end{array}$ \\
\hline 37 & $\begin{array}{c}\text { Self-Efficacy about } \\
\text { leisure time physical } \\
\text { activity }\end{array}$ & Leisure & $C V I>0.79$ & $\begin{array}{l}\text { Test-retest: }(r=0.73, n=62 \text {, } \\
P<0.005) \\
\text { Internal consistency }=0.89\end{array}$ \\
\hline 38 & $\begin{array}{l}\text { Self-regulation about } \\
\text { leisure time physical } \\
\text { activity }\end{array}$ & Leisure & $C V I=0.90$ & Internal consistency=0.84 \\
\hline 39 & $\begin{array}{l}\text { Sleep disturbance scale } \\
\text { for children }\end{array}$ & Sleep/Rest & $\begin{array}{l}\text { Convergent Validity: }(r=0.22- \\
0.76)\end{array}$ & Internal consistency $=0.82$ \\
\hline 40 & Sleep hygiene index & Sleep/Rest & All fit indices acceptable & $\begin{array}{c}\text { ICC }=0.85 \\
\text { Test-retest: }(r=0.86, P<0.01)\end{array}$ \\
\hline 41 & $\begin{array}{l}\text { Adolescent sleep } \\
\text { hygiene scale }\end{array}$ & Sleep/Rest & All fit indices acceptable & $\begin{array}{c}\text { Internal consistency: } 0.71- \\
.0 .79 \\
\text { Test-retest: } 0.82-0.87\end{array}$ \\
\hline 42 & $\begin{array}{l}\text { Specific nordic ques- } \\
\text { tionnaire }\end{array}$ & Work & $\begin{array}{l}\text { Construct validity: }(r=0.72, \\
\qquad P<0.05)\end{array}$ & Test-retest: $\mathrm{k}=0.83$ \\
\hline 43 & $\begin{array}{l}\text { Task self-efficacy scale } \\
\text { for everyday activities }\end{array}$ & $A D L$ & All fit indices acceptable & $\begin{array}{c}\mathrm{ICC}=0.83 \\
\text { Internal consistency }=0.98\end{array}$ \\
\hline 44 & $\begin{array}{c}\text { Social participation } \\
\text { questionnaire }\end{array}$ & Social participation & Content validity $>0.70$ & Internal consistency $>0.7$ \\
\hline
\end{tabular}




\begin{tabular}{|c|c|c|c|c|}
\hline No & Measure & $\begin{array}{l}\text { Occupational Therapy Practice } \\
\text { Framework Dimensions }\end{array}$ & Validity & Reliability \\
\hline 45 & $\begin{array}{l}\text { Verran and snyder- } \\
\text { halpern sleep scale }\end{array}$ & Sleep/Rest & CVR $>0.62$ & $\begin{array}{c}\text { ICC }=0.69 \\
\text { Internal consistency }=0.83\end{array}$ \\
\hline 46 & $\begin{array}{l}\text { Walton's quality of } \\
\text { work-life }\end{array}$ & Work & All fit indices acceptable & Internal consistency: 0.89 \\
\hline 47 & $\begin{array}{l}\text { Wheelchair outcome } \\
\text { measure }\end{array}$ & ADL & $\begin{array}{l}\mathrm{CVR}=0.8 \\
\mathrm{CV} I>0.7\end{array}$ & $\begin{array}{c}\text { ICC }=0.99 \\
\text { Test-retest }=0.91\end{array}$ \\
\hline 48 & $\begin{array}{l}\text { Work ability index } \\
\text { questionnaire }\end{array}$ & Work & Criterion validity= 0.79 & $\begin{array}{l}\text { ICC }=0.92 \\
\text { Internal consistency }=0.79\end{array}$ \\
\hline 49 & $\begin{array}{l}\text { World health organiza- } \\
\text { tion health and work } \\
\text { performance question- } \\
\text { naire }\end{array}$ & Work & All fit indices acceptable & Internal consistency $>0.73$ \\
\hline 50 & $\begin{array}{l}\text { Work-related low back } \\
\text { pain predictor ques- } \\
\text { tionnaire }\end{array}$ & Work & All fit indices acceptable & $\begin{array}{l}\text { Internal consistency: } 0.75- \\
0.85\end{array}$ \\
\hline
\end{tabular}

Пranian Rehabilitation 】ournal

ADL: Activities of Daily Living; IADL: Instrumental Activities of Daily Living; CVI: Content Validity Index; ICC: Intraclass Correlation Coefficient; CVR: Content Validity Ratio.

All fit indices such as Kaiser-Meyer-Olkin and Bartlett's test of sphericity were acceptable

Table 2 lists 50 extracted measures. A total of 44 of the scales were translated into Persian and 6 were established for the Iranian population. The number of items in the included measures ranged from 5-197.

Table 3 presents the psychometric properties of the assessment measures and the "Framework" dimensions that they cover (Most studies reported on the validation of measures). Different types of validity (content, construct, criteria, convergent, and divergent validity) and reliability (inter- and intra-rater and internal consistency) were assessed for measures. Most of the measures assess just one occupational area and 14 evaluate 2 or more.

\section{Discussion}

In comparison to the majority of systematic reviews, which emphasize the psychometric properties of measures, the present study analyzed the content of the measures. This helped to recognize the strength and limitations of these measures, as well as the development of a more efficient measure. It also provided a source of available participation measures in Persian for clinicians and researchers.

This study revealed that out of 50 measures, which evaluate the different aspects of participation available in Persian, only the following 6 measures have developed based on Iranian culture and have acceptable psychometric properties: “Iranian Children's Participation Assessment Scale", "Iranian Male Adolescents Outcome
Expectation about Leisure Time Physical Activity", "Self-regulation about Leisure time Physical Activity", "Self-efficacy about Leisure Time Physical Activity", "Work-related Low Back Pain Predictor Questionnaire", and "Social Participation Questionnaire" (Table 3).

The rest were developed in other countries and translated to Persian. It is essential to consider cultural differences when using any assessment developed in another country. Although international standards for translating some measures (e,g., "Sleep Hygiene Index" and "Occupational Gaps Questionnaire") have been respected and cultural differences have been investigated, in many cases, such as "Children Assessment of Participation and Environment" and "Assessment of Life Habits", it is weakly considered.

It is recommended to Iranian therapists to develop or revise existing assessments to more fully measure the problems and needs of Iranians. Assessments should measure occupational participation and occupational performance based on the culture, in which the assessment is used. Borrowing assessments from another country, culture, or profession always result in some limitations of effectiveness and efficiency. Concepts can be borrowed, but they must be interpreted concerning the people's culture being assessed. As a result, assessing cross-cultural validation for translated measures is suggested.

The "Occupational Therapy Practice Framework: Domain and Process" provides a comprehensive view of occupational areas. Considering the importance of ac- 
curate and inclusive assessment of this construct, it was decided to use the last version (third edition) as a framework for reviewing the available participation measures in Persian. Measures that contained at least once about each of the "Framework" domains, were identified. As presented in Table 2, only the Iranian children's participation assessment scale, which developed based on the "Framework", covers all domains of participation.

Ignoring the aspects of occupations may limit the measure usefulness as a measure of individuals' reported participation. Therapists should consider the occupational areas in selecting an appropriate assessment tool. Therefore, it is suggested that although it is costly and time-consuming, researchers develop assessment tools that thoroughly cover occupational areas based on our culture in different populations.

As the "Framework" was created for the practice of occupational therapy in the United States, any use of the document outside the United States must acknowledge that the document does not account for other cultures or cultural differences. It is recommended that Iranian therapists write a document based on the "Framework" considering Iranian values and beliefs regarding participation in occupation in Iranian society and best approaches to assessment and intervention as applicable in rehabilitation programs in Iran.

It is highly important to consider psychometric properties (validity and reliability) of the measure while selecting the most appropriate one to evaluate efficacy and change over time. Almost all measures were evaluated in terms of some forms of reliability (e.g., internal consistency, inter- and intra-rater reliability) or validity (e.g., content, construct, convergent, and divergent validity).

Most of the measures offered only a Cronbach's alpha, which provides information on how well items within a measures group together (e,g., Insomnia Severity Index, Iranian Male Adolescent's Outcome Expectation about Leisure Time Physical Activity, and Independency Scale of Activities of Daily Living in Table 3). Problematically, Cronbach's alpha does not provide evidence of repeatability. There are several approaches to estimate reliability, such as test-retest and inter-rater reliability, which provide this type of evidence. This information needs more investment in data collection, but it is worthy to assess these types of reliability.

In the same way, most validity evidence came from the study of content validity. Structural validity (the degree, to which scores of a questionnaire are an adequate reflection of the dimensionality of the construct to be measured) and criterion validity (the degree, to which the scores of a health-related patient-reported outcomes measure are an adequate reflection of a "gold standard") require greater sampling to administer the measure in multiple groups or to collect a gold standard measure [75]. Few studies have investigated the former type of validity (e.g., community integration questionnaire, Craig hospital inventory of environmental factors, and Epworth sleepiness scale in Table 3). Multiple strategies should be included for a comprehensive assessment of the psychometric properties of a measure. These require researchers to allocate more time to measure development by gathering data across multiple time points, in multiple samples, or along with a gold standard.

Additionally, it is highly important to note that the quality of a measure and methodological quality of the study, which assessed the psychometric properties of the measure, are two different issues. If the methodological quality of the study is inadequate, the results cannot be trusted and the quality of the measure under study remains unclear. Terwee et al. developed criteria for good measurement properties that can guide occupational therapists in assessing the quality of the measure [76]. On the other hand, in recent times, an international Delphi study was done to develop the Consensus-based Standards for the selection of health Measurement Instruments (COSMIN) checklist for evaluating the methodological excellence of studies on measurement properties.

The COSMIN taxonomy and checklist can help the researchers to identify the need for further research on measurement properties. Researchers can also use the COSMIN checklist in designing their study to make sure that it meets the standards for excellent quality $[75,77]$.

Another important consideration when selecting a measure is its application for the target population. Measures developed for one setting or a special health condition should only be generalized with caution. The reliability and validity of most of the measures included in this review were assessed in certain populations, where their applicability in other groups remains questionable and needs more investigation. Limited access to the full text of some articles was a limitation of the present study. Because of the high number of measures identified, it was impossible to assess all of them based on the COSMIN checklist. It is, therefore, recommended to conduct future studies on assessing the measures based on the COSMIN checklist. 
Implications to practice, research, and education

This study by reviewing general characteristics (e,g., content, number of items, time, and format of administration) and psychometric properties of Persian participation measures in different occupational areas will help Persian-speaking therapists to select appropriate measures for assessing participation in occupational areas based on their resources.

Since the comprehensive assessment of occupational areas is crucial for enabling people in achieving occupational balance, clinicians and researchers can use the framework as a guide in selecting or developing measures. Also, measuring occupation from a culturally sensitive perspective is particularly important.

It also helps Persian-speaking researchers identify the weakness of existing measures and guide them in improving them or designing new ones. It is recommended that researchers can use the COSMIN checklist as a tool for this purpose. The COSMIN checklist helps researches to: 1. identify which measurement properties are relevant for evaluating health-related patient-reported outcomes; 2. introduce terminology and definitions of these measurement properties; and 3. identify the design requirements and preferred statistical methods. Accordingly, adding the COSMIN taxonomy and checklist and measurement properties criteria proposed by Terwee et al. in assessment and evaluation courses can highly improve rehabilitation education and assessment processes.

\section{Conclusion}

Most of the available measures were developed in other countries and translated into Persian. Only a few developed measures based on Iranian culture included all participation in occupational domains. Since the comprehensive assessment of occupational areas from a cultural perspective is crucial for enabling people in achieving occupational balance, this critical review will help Persian-speaking therapists to select appropriate measures for assessing participation in different occupational areas. Considering the lack of comprehensive measures for assessing participation in different ages, developing measures based on cultural properties seems necessary.

\section{Ethical Considerations}

\section{Compliance with ethical guidelines}

The systematic review was performed according to the PRISMA (Preferred Reporting Items for Systematic Reviews and Meta-Analyses) guidelines.

\section{Funding}

The research has not received any funding.

\section{Authors' contributions}

Data acquisition: Ghodsiyeh Joveini; Reviewing and revising the manuscript critically: Mitra Khalafbeigi; Critical analysis and Data analysis: Ghodsiyeh Joveini, Laleh Lajevardi, and Armin Zareiyan; Developing the concept, Reviewing extracted articles, Extracting the measures: Ghodsiyeh Joveini, Laleh Lajevardi.

\section{Conflict of interest}

The authors declared no conflict of interest.

\section{Acknowledgments}

We would like to express our gratitude to Dr. Afsoon Hassani Mehraban, Ph.D of Occupational Therapy, Iran University of Medical Sciences, for sharing her knowledge in this research.

\section{References}

[1] American Occupational Therapy Association (AOTA). Occupational therapy practice framework: Domain \& process ( $3^{\text {rd }}$ edition). American Journal of Occupational Therapy. 2017; 68:S1-48. [DOI:10.5014/ajot.2014.682006]

[2] Hammell KW. Quality of life, participation and occupational rights: A capabilities perspective. Australian Occupational Therapy Journal. 2015; 62(2):78-85. [DOI:10.1111/14401630.12183] [PMID]

[3] Law M, King G, King S, Kertoy M, Hurley P, Rosenbaum $\mathrm{P}$, et al. Patterns of participation in recreational and leisure activities among children with complex physical disabilities. Developmental Medicine and Child Neurology. 2006; 48(5):337-42. [DOI:10.1017/S0012162206000740] [PMID]

[4] Larson RW, Verma S. How children and adolescents spend time across the world: work, play, and developmental opportunities. Psychological Bulletin. 1999; 125(6):701-36. [DOI:10.1037/0033-2909.125.6.701] [PMID]

[5] Passmore A. The occupation of leisure: Three typologies and their influence on mental health in adolescence. OTJR: Occupation, Participation and Health. 2003; 23(2):76-83. [DOI:10.11 77/153944920302300205]

[6] Rezende MP, Tedeschi Cano MA, Chaves Mauro MY, de Oliveira DC, Marziale MHP, do Carmo Cruz Robazzi ML. Occupations performed by adolescents and their relationship with school participation. Acta Paulista de Enfermagem. 2012; 25(6):873-8. [DOI:10.1590/S0103-21002012000600008] 
[7] de Róiste A, Kelly C, Molcho M, Gavin A, Gabhainn SN Is school participation good for children? Associations with health and wellbeing. Health Education. 2012; 112(2):88-104. [DOI:10.1108/09654281211203394]

[8] Kvam L, Vik K, Eide AH. Importance of participation in major life areas matters for return to work. Journal of Occupational Rehabilitation. 2015; 25(2):368-77. [DOI:10.1007/ s10926-014-9545-2] [PMID] [PMCID]

[9] Whiteford G. Occupational deprivation: Global challenge in the new millennium. British Journal of Occupational Therapy. 2000; 63(5):200-4. [DOI:10.1177/030802260006300503]

[10] Westhorp P. Exploring balance as a concept in occupational science. Journal of Occupational Science. 2003; 10(2):99-106. [DOI:10.1080/14427591.2003.9686516]

[11] Stevelink S, Van Brakel W. The cross-cultural equivalence of participation instruments: A systematic review. Disability and Rehabilitation. 2013; 35(15):1256-68. [DOI:10.3109/096382 88.2012.731132] [PMID]

[12] Imms C. Review of the children's assessment of participation and enjoyment and the preferences for activity of children. Physical \& Occupational Therapy in Pediatrics. 2008; 28(4):389-404. [DOI:10.1080/01942630802307135] [PMID]

[13] Coster W, Bedell G, Law M, Khetani MA, Teplicky R, Liljenquist K, et al. Psychometric evaluation of the participation and environment measure for children and youth. Developmental Medicine \& Child Neurology. 2011; 53(11):1030-7. [DOI:10.1111/j.1469-8749.2011.04094.x] [PMID]

[14] Eriksson G, Tham K, Kottorp A. A cross-diagnostic validation of an instrument measuring participation in everyday occupations: The Occupational Gaps Questionnaire (OGQ). Scandinavian Journal of Occupational Therapy. 2013; 20(2):152-60. [DOI:10.3109/11038128.2012.749944] [PMID]

[15] Taheri Tanjani P, Azadbakht M. [Psychometric properties of the Persian version of the activities of daily living scale and instrumental activities of daily living scale in elderly (Persian)]. Journal of Mazandaran University of Medical Sciences. 2016; 25(132):103-12. http://jmums.mazums.ac.ir/article1-6766-en.html

[16] Dehghan SK, Rassafiani M, Akbar Fahimi N, Farahbod M Salehi M. [Validity and reliability of Activities Scale for Kids (ASK) in children with cerebral palsy (Persian)]. Journal of Research in Rehabilitation Sciences. 2011; 7(3):267-77. http:/ / jrrs.mui.ac.ir/index.php/jrrs/article/view/182

[17] Oveisgharan S, Shirani S, Ghorbani A, Soltanzade A, Baghaei A, Hosseini S, et al. Barthel index in a Middle-East country: Translation validity and reliability. Cerebrovascular Diseases. 2006; 22(5-6):350-4. [DOI:10.1159/000094850] [PMID]

[18] Javadi M, Javadi A, Kalantari N, Jaliloghadr Sh, Mohamad H. Sleep problems among pre-school children in Qazvin Iran. The Malaysian Journal of Medical Sciences. 2014; 21(6):52-6. [PMID] [PMCID]

[19] Dehghan L, Dalvand H, Pourshahbaz A. [Translation of Canadian occupational performance measure and testing Persian version validity and reliability among Iranian mothers of children with cerebral palsy (Persian)]. Journal of Modern Rehabilitation. 2015; 9(4):25-31. http://mrj.tums.ac.ir/ article-1-5349-en.html
[20] Amirian SR, Rezaee M, Pashazadeh Azari Z, Tabatabaee SM. [Validity and reliability of children's assessment of participation and enjoyment for people with disability aged 7-17 years old (Persian)]. Scientific Journal of Rehabilitation Medicine. 2015; 4(1):26-32. http:// medrehab.sbmu.ac.ir/article_1100005.html

[21] Ozgoli G, Sheikhan Z, Soleimani F, Nasiri M, Amiri S. Prevalence of sleep disorders among children 4-6 years old in Tehran Province Iran. Iranian Red Crescent Medical Journal. 2016; 18(7):e22052 [DOI:10.5812/ircmj.22052] [PMID] [PMCID]

[22] Dabiri Golchin M, Mirzakhani N, Stagnitti K, Dabiri Golchin M, Rezaei M. Psychometric properties of Persian version of "child-initiated pretend play assessment" for Iranian children. Iranian Journal of Pediatrics. 2016; 27(1):e7053. [DOI:10.5812/ijp.7053]

[23] Mirzakhani N, Dabiri Golchin M, Rezaee M, Tabatabaee SM, Dabiri Golchin M, Stagnitti K, et al. [Relaibility of Persian version of ChIPPA for pretend play assessment in children (Persian)]. Pejouhandeh. 2016; 21(2):87-92. http://pajoohande.sbmu.ac.ir/article-1-2174-en.html

[24] Amini M, Hassani Mehraban A, Rostamzade O. [Translation, cultural adaptation, and face, content, and convergent validity of children participation questionnaire into Persian (Persian)]. Journal of Rehabilitation Medicine. 2016; 5(2):1517. https:// www.researchgate.net/publication/315837765

[25] Amini M, Hassani Mehraban A, Rostamzadeh O, Mehdizadeh F. Psychometric properties of the Iranian-Children Participation Questionnaire (I-CPQ) when used with parents of preschool children with cerebral palsy. Occupational Therapy in Health Care. 2017; 31(4):341-51. [DOI:10.1080/07380577.20 17.1382753] [PMID]

[26] Negahban H, Fattahizadeh P, Ghasemzadeh R, Salehi R, Majdinasab N, Mazaheri M. The Persian version of community integration questionnaire in persons with multiple sclerosis: Translation reliability validity and factor analysis. Disability and Rehabilitation. 2013; 35(17):1453-9. [DOI:10.3109/ 09638288.2012.741653] [PMID]

[27] Nobakht Z, Rassafiani M, Reza Soltani P. Validity and reliability of Persian version of Craig Hospital Inventory of Environmental Factors (CHIEF) in children with cerebral palsy. Iranian Rehabilitation Journal. 2011; 9(1):3-10. http:// irj.uswr.ac.ir/article-1-199-en.html

[28] Sadeghniiat Haghighi Kh, Montazeri A, Khajeh Mehrizi A, Aminian O, Rahimi Golkhandan A, et al. The Epworth Sleepiness Scale: Translation and validation study of the Iranian version. Sleep and Breathing. 2013; 17:419-26. [DOI:10.1007/ s11325-012-0646-x] [PMID]

[29] Dehnadi-Moghadam A, Rezaei S, Khodadadi N, Rahmatpour P. Psychometric properties of the Functional Independence Measure (FIM) in Iranian patients with traumatic brain injury. Trauma Monthly. 2017; 22(1):e25534. [DOI:10.5812/ traumamon.25534]

[30] Naghdi S, Nakhostin Ansari N, Raji P, Shamili A, Amini $M$, Hasson S. Cross-cultural validation of the Persian version of the Functional Independence Measure for patients with stroke. Disability and Rehabilitation. 2016; 38(3):289-98. [DO I:10.3109/09638288.2015.1036173] [PMID]

[31] Rezaei S, Dehnadi Moghadam A, Khodadadi N, Rahmatpour P. Functional independence measure in Iran: A con- 
firmatory factor analysis and evaluation of ceiling and floor effects in traumatic brain injury patients. Archives of Trauma Research. 2015; 4(4):e25363. [DOI:10.5812/atr.25363]

[32] Razazian N, Najafi F, Mahdavi P, Aghaei A. [Prevalence of sleep disorders in patients with multiple sclerosis (Persian)]. Journal of Mazandaran University of Medical Sciences. 2014; 23(110):219-24. http://jmums.mazums.ac.ir/article-1-3384-en.html

[33] Fallahpour M, Jonsson H, Joghataei MT, Kottorp A. Impact on Participation and Autonomy (IPA): Psychometric evaluation of the Persian version to use for persons with stroke. Scandinavian Journal of Occupational Therapy. 2011; 18(1):59-71. [DOI:10.3109/11038121003628353] [PMID]

[34] Vazirinejad R, Joorian J, Taghavi MM, Lilley JM, Sayadi Anari AR. The Persian Version of a Participation Scale: Is it valid and reliable enough for use among Iranian patients with multiple sclerosis? Journal of Clinical Neurology. 2015; 11(2):157-63. [DOI:10.3988/jcn.2015.11.2.157] [PMID] [PMCID]

[35] Safa A, Masoudi Alavi N, Abedzadeh-Kalahroudi M. Predictive factors of dependency in activities of daily living following limb trauma in the elderly. Trauma Monthly. 2016; 21(5):e25091. [DOI:10.5812/traumamon.25091] [PMID] [PMCID]

[36] Sadeghniiat-Haghighi K, Montazeri A, Khajeh-Mehrizi A, Nedjat S, Aminian O. The Insomnia Severity Index: Cross-cultural adaptation and psychometric evaluation of a Persian version. Quality of Life Research. 2014; 23(2):533-7. [DOI:10.1007/s11136-013-0489-3] [PMID]

[37] Amini M, Hassani Mehraban A, Haghni H, Asgharnezhad AA, Khayatzadeh Mahani M. Development and validation of Iranian children's participation assessment scale. Medical Journal of the Islamic Republic of Iran. 2016; 30:333. [PMID] [PMCID]

[38] Amini M, Hassani Mehraban A, Haghani H, Mollazade E, Zaree M. Factor structure and construct validity of Children Participation Assessment Scale in activities outside of School-Parent version (CPAS-P). Occupational Therapy In Health Care. 2017; 31(1):44-60. [DOI:10.1080/07380577.2016.1272733] [PMID]

[39] Mohammadi A, Hassani Mehraban A, Ansari Damavandi Sh, Alizadeh Zarei M, Amini M. Participation in daily life activities among children with cancer. Middle East Journal of Cancer. 2017; 8(4):213-22. http:// mejc.sums.ac.ir/article_42092.html

[40] Abasi MH, Eslami AA, Rakhshani F. Introducing an outcome expectation questionnaire and its psychometric properties regarding leisure time physical activity for Iranian male adolescent. Iranian Red Crescent Medical Journal. 2015; 17(5):e21509. [DOI:10.5812/ircmj.21509] [PMID] [PMCID]

[41] Vasheghani-Farahani A, Tahmasbi M, Asheri H, Ashraf H, Nedjat S, Kordi R. The Persian, last 7-day, long form of the International Physical Activity Questionnaire: Translation and validation study. Asian Journal of Sports Medicine. 2011; 2(2):106-16. [DOI:10.5812/asjsm.34781] [PMID] [PMCID]

[42] Baghiani Moghaddam MH, Bakhtari Aghdam F, Asghari Jafarabadi M, Allahverdipour H, Dabagh Nikookheslat S, Safarpour Sh. The Iranian version of International Physical Activity Questionnaire (IPAQ) in Iran: Content and construct validity, factor structure, internal consistency and stability. World Applied Sciences Journal. 2012; 18(8):1073-80. https://www.researchgate.net/publication/286407389

[43] Tabatabaee Jabali SM, Ghaffari M, Pournik O, Ghalichi L, Tehrani Yazdi AR, Motevalian SA. Reliability and validity of Persian version of job content questionnaire in health care workers in Iran. The International Journal of Occupational and Environmental Medicine. 2013; 4(2):96-101. https:/ / www.theijoem.com/ijoem/index.php/ijoem/article/view/144/362

[44] Alizadeh Khoei M, Esmail Akbari M, Sharifi F, Fakhrzadeh $\mathrm{H}$, Larijani B. Translation and validation of the activities of daily living scale with Iranian elderly cancer patients treated in an oncology unit. Asian Pacific Journal of Cancer Prevention. 2013; 14(5):2731-7. [DOI:10.7314/APJCP.2013.14.5.2731] [PMID]

[45] Hassani Mehraban A, Soltanmohamadi Y, Akbarfahimi M, Taghizadeh Gh. Validity and reliability of the Persian version of Lawton instrumental activities of daily living scale in patients with dementia. Medical Journal of The Islamic Republic of Iran. 2014; 28:25. [PMID] [PMCID]

[46] Soltanmohamadi Y, Hassani Mehraban A, Taghizade Gh, Akbarfahimi M, Alahyari F. [Validity and reliability of the Persian version of Lawton instrumental activities of daily living scale among patients with dementia (Persian)]. Salmand: Iranian Journal of Ageing. 2014; 9(2):160-7. http:/ / salmandj. uswr.ac.ir/article-1-638-en.html

[47] Mortazavi SN, Rezaei M, Rassafiani M, Tabatabaei SM, Mirzakhany N, Sahaf R. [Validity and reliability of Persian version of LIFE Habits assessment for children with cerebral palsy aged between 5 and 13 years old (Persian)]. Archives of Rehabilitation. 2014; 14(S2):115-23. http://rehabilitationj. uswr.ac.ir/article-1-1432-en.html

[48] Delshad M, Ghanbarian A, Rezaei Ghaleh N, Amirshekari G, Askari S, Azizi F. Reliability and validity of the modifiable activity questionnaire for an Iranian urban adolescent population. International Journal of Preventive Medicine. 2015; 6:3. [DOI:10.4103/2008-7802.151433] [PMID] [PMCID]

[49] Momenan AA, Delshad M, Sarbazi N, Rezaei Ghaleh N, Ghanbarian A, Azizi F. Reliability and validity of the Modifiable Activity Questionnaire (MAQ) in an Iranian urban adult population. Archives of Iranian Medicine. 2012; 15(5):279-82. [PMID]

[50] Tagharrobi Z, Sharifi Kh, Sooky Z. [Psychometric evaluation of Shah version of modified Barthel index in elderly people residing in Kashan Golabchi nursing home (Persian)]. Feyz. 2011; 15(3):213-24. http:// feyz.kaums.ac.ir/article1-1241-en.html

[51] Fallahpour M, Tham K, Joghataei MT, Jonsson H. Perceived participation and autonomy: Aspects of functioning and contextual factors predicting participation after stroke. Journal of Rehabilitation Medicine. 2011; 43(5):388-97. [DOI:10.2340/16501977-0789] [PMID]

[52] Eftekharian R, Kaldi AR, Sum Sh, Sahaf R, Fadaye Vatan R. [Validity and reliability of Persian version of Onyx Social Capital Scale in elderly people (Persian)]. Salmand: Iranian Journal of Ageing. 2016; 11(1):174-89. [DOI:10.21859/sija1101174]

[53] Azadi HR, Tahmasbi A. [The study of reliability of performance assessment of self-care skills in evaluating the self-care skills of adult patients suffering from multiple sclerosis in Tehran (Persian)]. Archives of Rehabilitation. 2014; 15(3):6471. http:/ / rehabilitationj.uswr.ac.ir/article-1-1326-en.html

[54] Moradi Abbasabadi M, Akbarfahimi N, Hosseini SA, Rezasoltani P. [Reliability of the Persian version of the pediatric evaluation of disability inventory in 3 to 9-year old children with cerebral palsy (Persian)]. Journal of Mazandaran University of Medical Sciences. 2015; 25(130):129-37. http://jmums. mazums.ac.ir/article-1-6402-en.html 
[55] Nazifi M, Mokarami HR, Akbaritabar AA, Kalte HO, Rahi A. Psychometric properties of the Persian translation of Pittsburgh Sleep Quality Index. Health Scope. 2014; 3(2):e15547. [DOI:10.17795/jhealthscope-15547]

[56] Farrahi J, Nakhaee N, Sheibani V, Garrusi B, Amirkafi A. Psychometric properties of the Persian version of the Pittsburgh Sleep Quality Index Addendum for PTSD (PSQI-A). Sleep and Breathing. 2009; 13(3):259. [DOI:10.1007/s11325008-0233-3] [PMID]

[57] Amiri P, Jalali-Farahani S, Zarkesh M, Barzin M, Kaviani R, Ahmadizad S. Reliability and validity of the Iranian version of the QAPACE in adolescents. Quality of Life Research. 2014; 23(6):1797-802. [DOI:10.1007/s11136-014-0625-8] [PMID]

[58] Negahban H, Masoudpur F, Rajaei E, Nazarinia MA, Mazaheri M, Salavati M. Reliability validity and responsiveness of the Persian version of the Rheumatoid and Arthritis Outcome Score (RAOS) in patients with rheumatoid arthritis. Clinical Rheumatology. 2015; 34:35-42. [DOI:10.1007/s10067-014-2515-4] [PMID]

[59] Farsi AR, Fathirezaie Z, Zamani-Sani SH. [Psychometrics of the recreation experience preference scale in Iranian elderly (Persian)]. Feyz. 2016; 19(6): 533-42. http:/ / feyz.kaums.ac.ir/ article-1-2923-en.html

[60] Kolagari Sh, Zagheri Tafreshi M, Rassouli M, Kavousi A Psychometric evaluation of the role strain scale: The Persian version. Iranian Red Crescent Medical Journal. 2014; 16(10):e15469. [DOI:10.5812/ircmj.15469] [PMID] [PMCID]

[61] Abasi MH, Eslami AA, Rakhshani F, Shiri M. A self-efficacy questionnaire regarding leisure time physical activity: Psychometric properties among Iranian male adolescents. Iranian Journal of Nursing and Midwifery Research. 2016; 21(1):20-8. [DOI:10.4103/1735-9066.174751] [PMID] [PMCID]

[62] Abasi MH, Eslami AA, Rakhshani F, Shiri M. Development and psychometric properties of a self-regulation scale about leisure time physical activity in Iranian male adolescents. Iranian Journal of Nursing and Midwifery Research. 2016; 21(2):183 90. [DOI:10.4103/1735-9066.178246] [PMID] [PMCID]

[63] Saffari M, Gholamrezaei A, Saneian H, Attari A, Bruni O. Linguistic validation of the Sleep Disturbance Scale for Children (SDSC) in Iranian children with Persian language. Sleep Medicine. 2014; 15(8):998-1001. [DOI:10.1016/j. sleep.2014.03.021] [PMID]

[64] Chehri A, Kiamanesh AR, Ahadi H, Khazaie H. [Psychometric properties of the Persian version of Sleep Hygiene Index in women (Persian)]. Journal of Kermanshah University of Medical Sciences. 2016; 19(6):e69794. [DOI:10.22110/jkums.v19i6.2677]

[65] Chehri A, Khazaie H, Eskandari S, Khazaie S, HolsboerTrachsler E, Brand S, et al. Validation of the Farsi version of the revised Adolescent Sleep Hygiene Scale (ASHSr): A crosssectional study. BMC Psychiatry. 2017; 17:408. [DOI:10.1186/ s12888-017-1578-6] [PMID] [PMCID]

[66] Namnik N, Negahban H, Salehi R, Shafizadeh R, Tabib SM Validity and reliability of Persian version of the Specific Nordic questionnaire in Iranian industrial workers. Work. 2016; 54(1):35-41. [DOI:10.3233/WOR-162268] [PMID]

[67] Golmohammadi B, Kashani V, Mokaberian M. [The psychometric properties of Persian version of task self-efficacy scale for everyday activities in older adults (Persian)]. Journal of Clinical Psychology. 2015; 7(2):87-100. [DOI:10.22075/ JCP.2017.2202]
[68] Afshani SAR, Janatifar A. [The comparative study of social participation between state and non-profit high school students in Yazd and its relevant factors (Persian)]. Journal of Applied Sociology. 2016; 27(3):73-96. [DOI:10.22108/ JAS.2016.20502]

[69] Mashayekhi F, Mirzai Saifabad R, Baghery P. [Validity and reliability of the Verran and Snyder-Halpern Sleep Scale in Iranian population (Persian)]. Journal of Mazandaran University of Medical Sciences. 2016; 25(132):200-9. http://jmums. mazums.ac.ir/article-1-6794-en.html

[70] Khaghanizadeh M, Ebadi A, Cirati Nair M, Rahmani M. [The study of relationship between job stress and quality of work life of nurses in military hospitals (Persian)]. Journal of Military Medicine. 2008; 10(3):175-84. http://militarymedj. ir/article-1-251-en.html

[71] Alimohammad S, Parvaneh Sh, Ghahari S, Saberi H, Yekaninejad MS, Miller WC. Translation and validation of the Farsi version of the Wheelchair Outcome Measure (WhOM-Farsi) in individuals with spinal cord injury. Disability and Health Journal. 2016; 9(2):265-71. [DOI:10.1016/j.dhjo.2015.09.004] [PMID]

[72] Abdolalizadeh M, Arastoo AA, Ghsemzadeh R, Montazeri A, Ahmadi K, Azizi A. The psychometric properties of an Iranian translation of the Work Ability Index (WAI) questionnaire. Journal of Occupational Rehabilitation. 2012; 22(3):4018. [DOI:10.1007/s10926-012-9355-3] [PMID]

[73] Pournik O, Ghalichi L, Tehrani Yazdi AR, Tabatabaee SM, Ghaffari M, Vingard E. Reliability and validity of Persian version of World Health Organization health and work performance questionnaire in Iranian health care workers. The international journal of occupational and environmental medicine. 2012; 3(1):33-8.

[74] Shojaei S, Tavafian SS, Jamshidi AR, Wagner J. A multidisciplinary work-related low back pain predictor questionnaire: Psychometric evaluation of Iranian patient-care workers. Asian Spine Journal. 2016; 10(3):501-8. [DOI:10.4184/ asj.2016.10.3.501] [PMID] [PMCID]

[75] Mokkink LB, Terwee CB, Patrick DL, Alonso J, Stratford PW, Knol DL, et al. The COSMIN checklist for assessing the methodological quality of studies on measurement properties of health status measurement instruments: An international Delphi study. Quality of Life Research. 2010; 19(4):539-49. [DOI:10.1007/s11136-010-9606-8] [PMID] [PMCID]

[76] Terwee CB, Prinsen CAC, Chiarotto A, Westerman MJ, Patrick DL, Alonso J, et al. COSMIN methodology for evaluating the content validity of patient-reported outcome measures: a Delphi study. Quality of Life Research. 2018; 27(5):1159-70. [DOI:10.1007/s11136-018-1829-0] [PMID] [PMCID]

[77] Mokkink LB, de Vet HCW, Prinsen CAC, Patrick DL Alonso J, Bouter LM, et al. COSMIN risk of bias checklist for systematic reviews of patient-reported outcome measures. Quality of Life Research. 2018; 27(5):1171-9. [DOI:10.1007/ s11136-017-1765-4] [PMID] [PMCID] 
